\title{
Multiple Renal Arteries in Live Donor Renal Transplantation; Impact on Graft Function and Outcome: A Prospective Cohort Study
}

\author{
Dilushi Rowena Wijayaratne1, Dinesha Himali Sudusinghe ${ }^{2,3}$, Nalaka Gunawansa ${ }^{4}$ \\ ${ }^{1}$ University Medical Unit, National Hospital of Sri Lanka, Colombo, Sri Lanka \\ ${ }^{2}$ National Hospital of Sri Lanka, Colombo, Sri Lanka \\ ${ }^{3}$ Department of Physiology, University of Sri Jayawardenapura, Nugegoda, Sri Lanka \\ ${ }^{4}$ National Institute of Nephrology, Dialysis and Transplant, Colombo, Sri Lanka \\ Email: nalakagunawansa@yahoo.co.uk
}

How to cite this paper: Wijayaratne, D.R., Sudusingha, D.H. and Gunawansa, N. (2018) Multiple Renal Arteries in Live Donor Renal Transplantation; Impact on Graft Function and Outcome: A Prospective Cohort Study. Open Journal of Organ Transplant Surgery, 8, 1-11.

https://doi.org/10.4236/ojots.2018.81001

Received: September 26, 2017

Accepted: January 15, 2018

Published: January 18, 2018

Copyright $\odot 2018$ by authors and Scientific Research Publishing Inc. This work is licensed under the Creative Commons Attribution International License (CC BY 4.0).

http://creativecommons.org/licenses/by/4.0/ (c) (i) Open Access

\begin{abstract}
Introduction: The presence of multiple renal arteries (MRA) in the donor allograft was once a contraindication to transplantation. Despite concerns about risks, these allografts are being increasingly used to overcome a shortage of renal donors. Objectives: To compare the outcomes of live-donor renal allografts with multiple and single renal arteries (SRA) in terms of overall ischemia times, early and late graft function, and vascular and urological complications. Methods: A prospective, non-randomized cohort study was conducted including all live donor renal transplants done by the Vascular and Transplant Unit of the National Institute of Nephrology Dialysis and Transplantation, Sri Lanka between March 2010 and March 2016. 312 recipients of live donor renal allografts were recruited to the study. Patients were divided into three groups: Group 1-SRA: single anastomosis $(\mathrm{n}=264,85 \%)$; Group 2-MRA: single conjoined anastomosis $(\mathrm{n}=39,12 \%)$; and Group 3-MRA: $\geq 2$ anastomoses $(n=9,3 \%)$. Results: Mean ischaemia times (donor clamping to graft reperfusion) in the three groups were 14, 21 and 17 minutes respectively. Failure to normalize creatinine within 72 hours was seen in 29/264 (11\%), $4 / 39(10.2 \%)$ and $1 / 9(11 \%),(P>0.05)$. Delayed graft function (attributable to severe rejection) occurred in only one patient who was from group 2. One-year graft survival among the groups was 243/264 (92\%), 35/39 (90\%) and 8/9 (89\%), $(P>0.05)$. One patient from groups 1 and 2 developed transplant renal artery stenosis. Two patients from group 1 needed stenting for ureteric stenosis. Conclusions: Donor grafts with MRA may be accepted safely with careful surgical reconstruction and close surveillance posttransplant.
\end{abstract}




\section{Keywords}

Multiple Renal Arteries, Live Donor Renal Transplant

\section{Introduction}

The presence of multiple renal arteries (MRA) in prospective renal donors was once considered a relative contraindication to live donor renal transplantation (LDRT). This presumed technical difficulty in anastomosis and potential for increased vascular and secondary urological complications resulted in many potential healthy donors being overlooked for more favourable renal anatomy in deceased donors or alternate live donors [1]. However, the increasing disparity between the escalating demand for renal transplantation and relatively stagnant supply of deceased donor organs has made it prohibitive for potential live donors to be declined based on arterial anatomy [2] [3]. Despite the technical difficulties and potential complications of transplanting donor kidneys with MRA, these organs are increasingly accepted to maximize the pool of acceptable donors [4]. With increasing expertise in vascular reconstruction and surgical technique, the acceptance rate of donors with MRA has increased universally among transplant centers. The impact of such complex donor arterial anatomy on the graft function and overall outcome remains an area of interest with limited available data.

The deceased donor program in Sri Lanka is still in its infancy with relatively poor donor rates compared to other countries. While definite forward strides have been made to increase deceased organ donation in the country over the last decade, LDRT presently remains the mainstay of transplantation in Sri Lanka. The National Institute of Nephrology Dialysis and Transplantation (NINDT) is currently the only dedicated transplant hospital in the country, and has been undertaking routine deceased and live donor transplants since 2010. This study was conducted by the Vascular and Transplant Unit of the NINDT to compare the short and mid-term outcomes of LDRT from accepted live donors having single renal arteries (SRA) and MRA.

\section{Methods}

We conducted a prospective cohort study of all LDRT performed between March 2010 and March 2016 by the Vascular and Transplant Unit of the NINDT. All successive adult ( $\geq 14$ years) LDRT performed by the unit during the study period were included. All paediatric ( $<14$ years) transplants and all adult deceased donor transplants were excluded. No live donors were declined on the basis of arterial anatomy during this period. The study population was divided in to two groups; MRA and SRA. The surgical unit comprised of a single specialist Transplant surgeon to perform the donor operation and a specialist Vascular and Transplant surgeon to perform the recipient operation. All recipient operations 
were performed by the same surgeon.

All live donor evaluations were done according to internationally accepted standard guidelines [5] [6]. All live donors underwent a rigorous medical, ethical and psychological evaluation based on the local guidelines and protocols laid out by the Ministry of Health, Sri Lanka. The side of donor nephrectomy was decided based on a combination of clinical parameters, renal arterial anatomy and differential renal function. Renal arterial anatomy was defined based on Computerised Tomographic Angiography. No donors were declined based on arterial anatomy during the study period. Most donor nephrectomies were performed by open surgery. A few hand-assisted laparoscopic nephrectomies were done where facilities were available. The choice was based mainly on availability of laparoscopic nephrectomy facilities in the hospital. All such laparoscopic nephrectomies were of kidneys with SRA. The donor and recipient operations were always carried out concurrently in adjoining operating theatres. Histidine tryptophan Ketogluterate (HTK) solution was used in all transplants for back-table flushing of the kidney and storage until recipient anastomosis.

Patients were divided into three groups: Group 1; single artery single anastomosis, Group 2; multiple arteries with single conjoined anastomosis, and Group 3: multiple arteries with two or more anastomoses. Whenever MRA were present, they were preferentially reconstructed on the "back-table" with side to side vertical spatulation to form a single opening using 7/0 polypropylene suture. All back-table reconstructions were carried out by the same specialist surgeon and although not included in the statistical analysis, the anastomotic time was kept below 10 minutes. Where the individual arteries were placed wide apart in the hilum not allowing a tension-free reconstruction, they were left for individual separate "on-table" anastomosis. Smaller capsular arteries that perfused less than $5 \%$ of the graft during back-table perfusion were ligated and not considered for reconstruction. In groups 1 and 2, the donor arteries were anastomosed to the recipient External Iliac Artery (EIA) in an end-to-side fashion using a continuous $6 / 0$ polypropylene suture. In group 3 , the anastomoses were done with the larger donor artery to the recipient EIA and the smaller donor artery to the recipient Inferior Epigastric Artery (IEA). The donor artery to IEA anastomosis was done in an end-to-end fashion with 7/0 interrupted polypropylene suture. The ureteric anastomosis was done to the recipient bladder to form a neoureterocystostomy in the standard Lich-Gregoir fashion over a 5 Fr ureteric stent [7]. All transplants received standardized immunosuppression comprising of basiliximab induction and prednisolone, tacrolimus and mycophenolate triple therapy posttransplant.

The transplant recipients were followed up by the surgical and nephrological teams at designated intervals. All recipients had routine duplex imaging of the graft within the first week of transplant and whenever delayed function was encountered. Repeat duplex imaging was done thereafter based on clinical findings and requirement.

All grafts were followed up and compared for possible graft dysfunction, ar- 
terial insufficiency and major urological complications. Delayed Graft Function (DGF) was defined as poor initial function after transplantation that required dialysis within the first week of the transplant. For the purposes of this study, any graft that demonstrated delayed function with failure to normalize serum creatinine levels within 72 hours of the transplant but not requiring haemodialysis were considered as slow graft function (SGF).

\section{Results}

A total of 312 live donor and 71 deceased donor transplants were carried out in our unit during the period of study. All 312 live donor recipients were recruited to the study, of whom 264 (85\%) had SRA and 48 (15\%) had MRA. The baseline characteristics of the study population is summarized in Table 1 . Among those with MRA, 41 had two, 5 had three and 2 had four arteries. Group 1 comprised of the 264 recipients of grafts with SRA. Group 2 had 39 patients and Group 3 had 9 patients. The mean overall ischaemia times, incidence of DGF or SGF, major urological complications, vascular complications, and 1-year graft survival are summarized in Table 2.

Table 1. Baseline characteristics.

\begin{tabular}{|c|c|c|c|}
\hline & $\begin{array}{c}\text { SRA } \\
\mathrm{N}=264\end{array}$ & $\begin{array}{c}\text { MRA } \\
\mathrm{N}=48\end{array}$ & $P$ value \\
\hline \multicolumn{4}{|l|}{ Gender } \\
\hline Male, number (\%) & $149(56 \%)$ & $32(67 \%)$ & 0.21 \\
\hline Female, number (\%) & $115(44 \%)$ & $16(33 \%)$ & \\
\hline \multicolumn{4}{|l|}{$\underline{\text { Age }}$} \\
\hline$<40 \mathrm{yrs}$ & $121(46 \%)$ & $19(39 \%)$ & 0.41 \\
\hline$\geq 40 \mathrm{yrs}$ & $143(54 \%)$ & $29(61 \%)$ & \\
\hline \multicolumn{4}{|l|}{ Co-morbidities } \\
\hline Diabetes & $197(74 \%)$ & $39(81 \%)$ & 0.37 \\
\hline Documented atherosclerotic arterial disease & $46(17 \%)$ & $05(10 \%)$ & 0.16 \\
\hline
\end{tabular}

$\mathrm{SRA}=$ Single renal artery, $\mathrm{MRA}=$ Multiple renal arteries.

Table 2. Short and Medium term outcomes of live-donor renal transplantation according to renal arterial anatomy of donor.

\begin{tabular}{|c|c|c|c|c|}
\hline Outcome & $\begin{array}{c}\text { Group 1 } \\
\text { SRA: Single anastomosis }(\mathrm{n}=264)\end{array}$ & $\begin{array}{c}\text { Group 2 } \\
\text { MRA: Single anastomosis }(\mathrm{n}=39)\end{array}$ & $\begin{array}{c}\text { Group 3 } \\
\text { MRA: >1 anastomosis }(\mathrm{n}=9)\end{array}$ & $P$ value \\
\hline $\begin{array}{l}\text { Mean ischaemia Time } \\
\left(\text { (minutes) }{ }^{*}\right.\end{array}$ & 14 & 21 & 17 & $P>0.05$ \\
\hline Delayed graft function & 0 & $1(0.03 \%)$ & 0 & $P>0.05$ \\
\hline Slow graft function & $29(11 \%)$ & $4(10 \%)$ & $1(11 \%)$ & $P>0.05$ \\
\hline Vascular complications & TRAS $1(0.003 \%)$ & TRAS $1(0.03 \%)$ & 0 & $P>0.05$ \\
\hline Urological complications & Ureteric stenosis $2(0.01 \%)$ & Ureteric dehiscence $1(0.03 \%)$ & 0 & $P>0.05$ \\
\hline 1 year graft survival & $243(92 \%)$ & 35 (90\%) & $8(89 \%)$ & $P>0.05$ \\
\hline
\end{tabular}

SRA—single renal artery, MRA—-multiple renal artery, $n$ —number, TRAS—-transplant renal artery stenosis; *donor arterial clamping to graft reperfusion. 
The ischaemia time in group 3 was less than in group 2, as the main artery was anastomosed first and graft reperfused while controlling the accessory artery with bull dog clamps. The accessory artery was then anastomosed while the rest of the kidney maintained perfusion. The ischaemia time in group 3 was less than in group 2, as the main artery was anastomosed first and graft reperfused prior to reconstructing the accessory artery. The mean follow-up was $16( \pm 4.8)$ months. DGF requiring haemodialysis within the first week was encountered only in one patient in the entire study group, and belonged to group 2 . This patient's DGF was a result of severe biopsy proven humoral rejection that required prolonged plasma exchange, intravenous immunoglobulin and rituximab therapy, thereby independent of the arterial anatomy. All patients with delayed function recovered graft function without the need for haemodialysis.

There were no instances of arterial insufficiency in the short term. There were two cases of transplant renal artery stenosis (TRAS) with resultant hypertension and graft dysfunction during continued follow up ( $9 \& 13$ months), one each in group 1 and 2 . Both patients were successfully managed by percutaneous renal artery angioplasty.

There were 2 cases of ureteric stenosis, both in group 1, presenting at 13 and 17 weeks post-transplant. Both patients had normal arterial graft perfusion on duplex imaging and the ureteric stenosis was successfully managed with a period of ureteric stenting. Surgical intervention was not required in these two patients. The patient in Group 2 with severe humoral rejection, discussed above, had complete ureteric dehiscence at approximately 8 weeks post-transplant with normal arterial graft perfusion. This was deemed unrelated to arterial inflow and a result of severe rejection and sustained heavy immunosuppression. The patency of both arteries in the graft was confirmed by pre-operative duplex as well as on-table assessment during ureteric re-implantation. The graft function was restored following treatment and the ureteric re-implantation was done.

\section{Discussion}

Although standard renal anatomy comprises of a single artery to each kidney arising from the aorta, the occurrence of MRA is a common anatomical variant. In one study by Pollak and colleagues (1986) that looked at 400 cadaveric donors, $28 \%$ had MRA, with $23 \%$ demonstrating double, $4 \%$ triple and $1 \%$ quadruple arteries [8]. In a smaller study by Munnusamy and colleagues (2016) looking at live donors in India, 38\% had MRA on pre-operative angiography; $13 \%$ on the right side, $13 \%$ on the left and $12 \%$ bilaterally [9]. This is comparable to other reports of MRA present unilaterally in $25 \%$ and bilaterally in $10 \%$ of the population.

Unlike renal veins which have multiple intrarenal anastomoses, allowing them to be ligated more freely, renal arteries are end arteries [10]. Ligation or injury to these arteries can render the supplied area ischemic, resulting in infarction. Therefore, reconstruction of all donor renal arteries other than small capsular branches is mandatory to obtain maximal graft outcome. The vascular anasto- 
mosis of renal grafts with MRA requires careful planning and reconstruction compared to the graft with a SRA. Whereas a SRA will only require a single anastomosis, grafts with MRA require either back-table reconstruction to make a common donor artery ostium or separate anastomosis of the individual arteries to the recipient [11] [12].

The arterial anastomosis in the recipient may be done to the common or EIA in an end-to-side fashion or to the internal iliac artery in an end-to-end fashion [13]. In our series, we practiced anastomosis to the recipient EIA as the standard practice in our unit. In cases of accessory donor arteries, the accessory artery can be reconstructed to the side of the main donor artery (Figure 1) or connected side by side with the main artery to form a common ostium [12]. This latter technique was used in all our patients having MRA and where the accessory arteries could reach the main artery without tension. Alternatively, the accessory artery can be anastomosed separately to the IEA or to a separate opening in the EIA. The sequence of arterial anastomosis and reperfusion in such dual anastomosis grafts remains an individual preference. While some surgeons prefer to complete both anastomosis prior to reperfusion, others perform the main anastomosis first, reperfuse the graft while controlling the accessory artery and then perform the second anastomosis while the graft remains perfused. This latter technique was practiced in our cohort of patients. In our series, wherever the accessory artery could not reach the main renal artery without tension, it was anastomosed in an end-to-end fashion to the IEA [14] [15]. The technique of

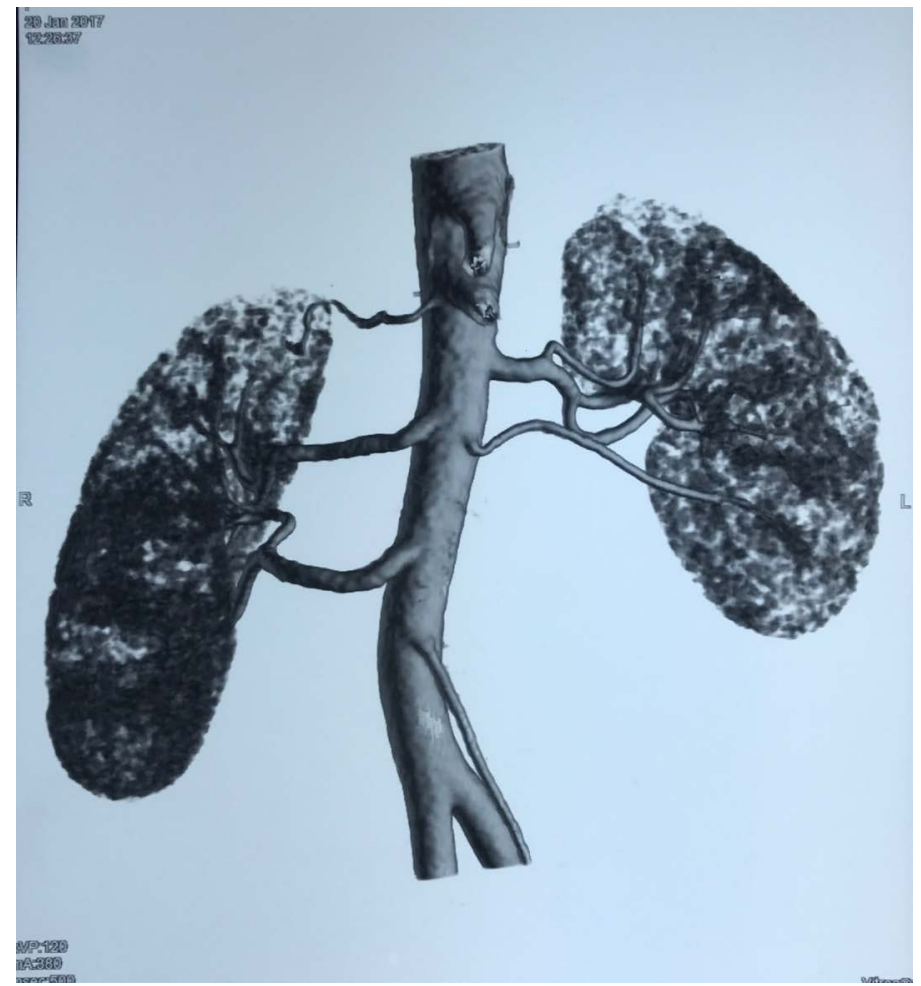

Figure 1. 3D reconstruction computerized tomography (CT) scan of a live renal donor depicting multiple renal arteries of both kidneys. 


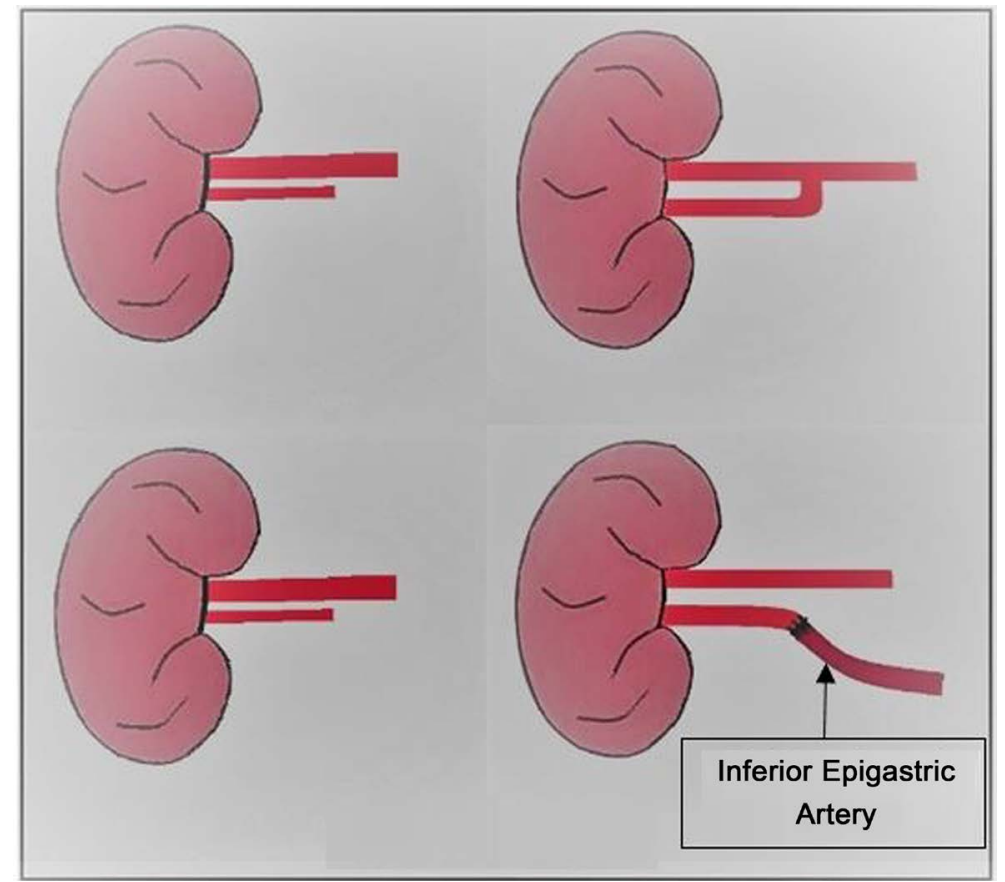

Figure 2. Schematic representation of the methods used in our study for vascular reconstruction of renal allografts with multiple renal arteries.

donor arterial reconstruction is dictated by the anatomy, quality of donor and recipient arteries and individual surgeons' preference.

In the case of deceased donors with MRA, all arteries may be removed along with a cuff of aorta (Carrel's patch), allowing for a single anastomosis in the recipient (Figure 2) [16]. While this renders the anastomosis technically easier, the resulting long arterial pedicle may predispose to kinking of the artery post-transplant [17]. In LDRT, inadequate length of donor vessels or other anastomotic difficulties can be circumvented by using recipient vessels such as the IEA, gonadal vein or great saphenous vein to reconstruct the relevant vessels. In the absence of suitable native vessels Polytetrafluoroethylene (PTFE) grafts have also been used successfully in reconstruction of graft vessels [18].

Both back-table reconstruction of the arteries and the creation of multiple in-situ arterial anastomoses prolong surgical ischemic times. However, extracorporeal reconstruction while in the cold preservation solution minimizes the warm ischaemia time while allowing for better visualization and easier suturing [19]. Alternatively, the main renal artery can be anastomosed to the recipient allowing earlier reperfusion of the graft while the accessory artery is kept under a soft clamp. This technique was used in our patients from group 3 . This again will minimize the ischaemic time to the major portion of the graft, allowing for subsequent accessory artery anastomosis while the rest of the graft is being perfused. Small capsular arteries which are less than $3 \mathrm{~mm}$ in diameter or are supplying $<10 \%$ of the graft kidney can be ligated safely with no clinically significant impact on graft function [16]. However, arteries supplying the lower pole of the kidney must be preserved and reconstructed to avoid compromising blood flow 
to the donor ureter [20] [21]. In our series, we attempted to reconstruct all arteries whenever possible and ligation of capsular arteries was done only where they were supplying the upper pole and $<5 \%$ of the kidney based on back-table perfusion assessment.

Apart from the intra-operative technical difficulties described above, concerns about grafts with MRA include the risk of vascular and urological complications post-transplant. Technical failure or post-anastomotic thrombosis, in particular of the lower polar arteries, can result in segmental infarction, infection, calyceal fistulae and ureteric necrosis or dehiscence. This may lead to increased morbidity and graft loss. However, Benedetti et al. (1995) demonstrated an incidence of post-operative renal artery thrombosis in 4 of 835 grafts with SRA while 163 grafts with MRA had no incidence of thrombosis [21].

Additional morbidities documented as being associated with MRA grafts include prolonged ischaemic time resulting in higher incidence of acute tubular necrosis, DGF and increased risk of rejection. MRA that require reconstruction have been thought to result in an increased incidence of TRAS with subsequent hypertension and possible reduced graft survival. For these reasons MRA was considered a relative contra-indication to kidney donation in the past [22]. The majority of these reservations were based on historical accounts of complications seen in deceased donor transplants. Better understanding of back-table arterial reconstruction, organ preservation with specialized preservation fluids and especially LDRT with minimal cold ischaemia times, have resulted in comparable results between grafts with SRA and MRA.

A systematic review and meta-analysis by Zorgdrager et al. (2016) comparing outcomes of recipients receiving MRA grafts or grafts with SRA showed higher rates of complications and DGF, as well as lower 1-year graft survival among patients with grafts with MRA [4]. However, this study looked at studies that included primarily deceased donor transplants and their outcomes. Rates of major urological complications in the MRA and SRA groups were 5.5\% and 5\% respectively. Vascular complications (arterial or venous thrombosis, TRAS, bleeding, hematoma, renal artery pseudo-aneurysm etc.) in the two groups were 10.8 and $8.1 \%$ respectively. Though statistically significant the pooled 1-year graft survival differed only mildly, being $93.2 \%$ in the MRA group and $94.5 \%$ in the SRA group. The long-term outcomes of 5-year graft survival and 1- and 5-year patient survival were similar in both groups. In our study of LDRT, we did not find any significant difference in outcomes between patients receiving grafts with SRA or MRA.

Other studies have not shown a difference in rates of acute tubular necrosis, biopsy proven acute rejection or overall graft survival in patients receiving grafts with SRA and MRA [21]. A study of 393 recipients by Makiyama et al. (2003) of grafts with MRA compared those who underwent reconstruction with those who did not [19]. As expected, total ischaemic times were longer in the reconstructed group. However, there was no difference in warm ischaemic times, early and late serum creatinine levels or acute rejection rates in the two groups. Post-transplant 
hypertension was commoner in the reconstructed group (68.2\% vs $48.6 \%$ ). This conforms with an earlier study that described an incidence of TRAS of $5.35 \%$ in a group with MRA undergoing reconstruction to form a single anastomosis compared to $1.4 \%$ in those with SRA an $1.9 \%$ in those with MRA with more than one anastomosis [21]. In our cohort, TRAS within the first year of follow up was encountered in one patient each in group 1 (SRA) and group 2 (MRA with a reconstructed ostium), which failed to reach statistical significance.

There are concerns about risks related to laparoscopic donor nephrectomy of grafts with MRA. In general, laparoscopic donor nephrectomy is preferred over open surgery as it is a less invasive procedure, avoids a large surgical scar, reduces post-operative pain and length of hospital stay [19]. There appear to be no differences between the donors with MRA and SRA undergoing laparoscopic nephrectomy in terms of serum creatinine and 1 year graft survival. A Korean study by Genc and colleagues (2011) comparing outcomes after laparoscopic donor nephrectomy for donor kidneys with one, two and three or more arteries found no difference in outcomes in terms of warm ischemia time and graft function [23]. In our cohort, laparoscopic nephrectomy was limited to 34 patients, all of whom had SRA. Limited expertise and facilities for laparoscopic donor nephrectomy in the public-sector health care system have limited its routine use in LDRT. However, with newer generation transplant surgeons with increased experience in laparoscopic live donor organ harvesting and expertise gained with the natural learning curve, we could look forward to laparoscopic nephrectomy extending in to all donor operations regardless of renal artery anatomy.

\section{Conclusion}

Recipients of grafts with MRA appear to have similar long-term outcomes compared to those of SRA. Although an increased incidence of perioperative vascular and urological complications has been observed in the past, this is largely limited to studies that looked at deceased donor grafts with numerous confounding variables such as increased cold ischaemia times and preservation damage. Nevertheless, the use of an aortic patch often circumvents any technical difficulty associated with deceased donor grafts having multiple arteries. In contrast, live donors having multiple arteries require careful planning and reconstruction. In the setting of a rising demand for renal allografts, donor grafts with MRA may be accepted safely with meticulous surgical reconstruction and close surveillance post-transplant. The observed short and medium-term graft outcomes show no significant difference to single artery grafts.

\section{References}

[1] Mazzucchi, E., Souza, A.A., Nahas, W.C., Antonopoulos, I.M., Piovesan, A.C. and Arap, S. (2005) Surgical Complications after Renal Transplantation in Grafts with Multiple Arteries. International Braz J Urol, 31, 125-130. https://doi.org/10.1590/S1677-55382005000200006

[2] Wetmore, J.B. and Collins, A.J. (2016) Global Challenges Posed by the Growth of 
End-Stage Renal Disease. Renal Replacement Therapy, 2, 15.

https://doi.org/10.1186/s41100-016-0021-7

[3] Moreno-Alarcón, C., Server-Pastor, G., López-González, P.Á., López-Cubillana, P., Ruiz-Morcillo, J.C., Doñate-Iñíguez, G., et al. (2013) Must We Still Be Worried about Multiple Arteries in Kidney Transplantation? Nephro-Urology Monthly, 5, 692-696. https://doi.org/10.5812/numonthly.4928

[4] Zorgdrager, M., Krikke, C., Hofker, S.H., Leuvenink, H.G.D. and Pol, R.A. (2016) Multiple Renal Arteries in Kidney Transplantation: A Systematic Review and Meta-Analysis. Annals of Transplantation, 21, 469-478. https://doi.org/10.12659/AOT.898748

[5] Delmonico, F. (2005) Council of the Transplantation Society. A Report of the Amsterdam Forum on the Care of the Live Kidney Donor: Data and Medical Guidelines. Transplantation, 79, S53-S66. https://doi.org/10.1097/01.TP.0000157343.27949.9F

[6] Abecassis, M., Adams, M., Adams, P., Arnold, R.M., Atkins, C.R., Barr, M.L., et al. (2000) Consensus Statement on the Live Organ Donor. JAMA, 284, 2919-2926. https://doi.org/10.1001/jama.284.22.2919

[7] Alberts, V.P., Idu, M.M., Legemate, D.A., Laguna Pes, M.P., Minnee, R.C., Mirza, C., et al. (2014) Ureterovesical Anastomotic Techniques for Kidney Transplantation: A Systematic Review and Meta-Analysis. Transplant International, 27, 593-605. https://doi.org/10.1111/tri.12301

[8] Pollak, R., Prusak, B.F. and Mozes, M.F. (1986) Anatomic Abnormalities of Cadaver Kidneys Procured for Purposes of Transplantation. Am Surg, 52, 233-235.

[9] Munnusamy, K., Kasirajan, S.P., Gurusamy, K., Raghunath, G., Bolshetty, S.L., Chakrabarti, S., et al. (2016) Variations in Branching Pattern of Renal Artery in Kidney Donors Using CT Angiography. Journal of Clinical and Diagnostic Research, 10, AC01-AC03. https://doi.org/10.7860/JCDR/2016/16690.7342

[10] Mir, N.S., Ul Hassan, A., Rangrez, R., Hamid, S., Sabia., Tabish, S.A., et al. (2008) Bilateral Duplication of Renal Vessels: Anatomical, Medical and Surgical Perspective. International Journal of Health Sciences, 2, 179-185.

[11] He, B., Mou, L., Mitchell, A. and Delriviere, L. (2013) Meticulous Use of Techniques for Reconstruction of Multiple Renal Arteries in Live Donor Kidney Transplantation. Transplantation Proceedings, 45, 1396-1398. https://doi.org/10.1016/j.transproceed.2012.12.013

[12] Berardinelli, L. (2006) Best Results in Living Donor Transplantation Using an Aggressive Policy in Microsurgical Bench Reconstruction of Nonoptimal Arterial Supply. Transplantation Proceedings, 38, 991-993. https://doi.org/10.1016/j.transproceed.2006.03.062

[13] Moray, G., Bilgin, N., Karakayali, H. and Haberal, M. (1999) Comparison of Outcome in Renal Transplant Recipients with Respect to Arterial Anastomosis: The Internal versus the External Iliac Artery. Transplantation Proceedings, 31, 2839-2840. https://doi.org/10.1016/S0041-1345(99)00584-9

[14] Mersa, B., Grkan, A., Zelik, B., Kaar, S., Varlsha, C., Turun, V., et al. (2011) Anastomosis of Accessory Arteries via Microsurgical Technique in Renal Transplantation. Transplantation Proceedings, 43, 819-821. https://doi.org/10.1016/j.transproceed.2011.01.114

[15] Antonopoulos, I.M., Yamaçake, K.G.R., Oliveira, L.M., Piovesan, A.C., Kanashiro, H. and Nahas W.C. (2014) Revascularization of Living-Donor Kidney Transplant with Multiple Arteries: Long-Term Outcomes Using the Inferior Epigastric Artery. Urology, 84, 955-959. https://doi.org/10.1016/j.urology.2014.06.022 
[16] Beckmann, J.H., Jackobs, S. and Klempnauer, J. (2008) Arterial Reconstruction in Kidney Transplantation. TX Med, 20, 7-12.

[17] Barry, J.M. (2007) Renal Transplant-Recipient Surgery. BJU International, 99, 701-717. https://doi.org/10.1111/j.1464-410X.2007.06785.x

[18] Tomic, A., Milovic, N., Marjanovic, I., Bjelanovic, Z., Lekovic, I., Mickovic, S., et al. (2015) Different Techniques of Vessel Reconstruction during Kidney Transplantation. Vojnosanitetski Pregled, 72, 614-618. https://doi.org/10.2298/VSP131210038T

[19] Makiyama, K., Tanabe, K., Ishida, H., Tokumoto, T., Shimmura, H., Omoto, K., et al. (2003) Successful Renovascular Reconstruction for Renal Allografts with Multiple Renal Arteries. Transplantation, 75, 828-832. https://doi.org/10.1097/01.TP.0000054461.57565.18

[20] Wolters, H.H., Schult, M., Heidenreich, S., Chariat, M., Senninger, N. and Dietl, K.H. (2001) The Anastomosis between Renal Polar Arteries and Arteria Epigastrica Inferior in Kidney Transplantation: An Option to Decrease the Risk of Ureter Necrosis? Transplant International, 14, 442-444. https://doi.org/10.1111/j.1432-2277.2001.tb00084.x

[21] Benedetti, E., Troppmann, C., Gillingham, K., Sutherland, D.E.R., Payne, W.D., Dunn, D.L., et al. (1995) Short- and Long-Term Outcomes of Kidney Transplants with Multiple Renal Arteries. Annals of Surgery, 221, 406-414.

https://doi.org/10.1097/00000658-199504000-00012

[22] Murray, J.E., Tilney, N.L. and Wilson, R.E. (1976) Renal Transplantation: A Twenty-Five Year Experience. Annals of Surgery, 184, 565-573.

https://doi.org/10.1097/00000658-197611000-00006

[23] Genc, V., Karaca, A.S., Orozakunov, E., Cakmak, A., Sevim, Y., Ustuner, E., et al. (2011) Multiple Renal Arteries Challenge in Laparoscopic Donor Nephrectomy: How Far Can We Go? Journal of the Korean Surgical Society, 80, 272-277. https://doi.org/10.4174/jkss.2011.80.4.272 